\title{
What do simple hematological parameters tell us in patients with systemic sclerosis?
}

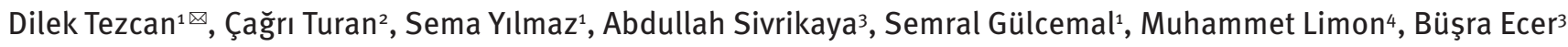

${ }^{1}$ Division of Rheumatology, Selçuk University, Faculty of Medicine, Selçuklu, Turkey. ${ }^{2}$ Department of Dermatology, Erzurum Education and Research Hospital, Erzurum, Turkey. ${ }^{3}$ Department of Biochemistry, Selçuk University, Faculty of Medicine, Selçuklu, Turkey. ${ }^{4}$ Division of Rheumatology, Kahramanmaraş Necip Fazıl City Hospital, Kahramanmaraş, Turkey.

\begin{abstract}
Introduction: Systemic sclerosis (SSc) or scleroderma is a clinically heterogeneous disease. Autoantibodies associated with different clinical features may help in predicting organ involvement. Complete blood count (CBC) parameters and neutrophil/lymphocyte (NLR), monocyte/lymphocyte (MLR), and platelet/lymphocyte (PLR) ratios, which are considered biomarkers of systemic inflammation, have been reported many times in various rheumatologic diseases. Studies related to the usefulness of the CBC to assess the severity of SSc are still lacking. This study seeks to determine whether CBC parameters associated with organ involvement, when evaluated together with clinical features and autoantibodies, can additionally contribute to risk estimation.

Methods: Adult patients with SSc $(n=130)$ and healthy control $(n=129)$ groups were enrolled in the study. Epidemiological, clinical, laboratory, and radiological findings were obtained by examining patient records.

Results: PLR, NLR, and MLR were related to organ involvement. Statistically significant results were obtained with hemoglobin ( $\leq$ $13.0 \mathrm{~g} / \mathrm{dl})$, lymphocyte count $\left(\leq 1,900 \times 10^{3} / \mathrm{ml}\right)$, and mean platelet volume $(\leq 8.0 \mathrm{fl})$ to estimate the risk of interstitial lung disease $(p<0.05)$. When the lymphocyte count was $1,400\left(10^{3} / \mathrm{ml}\right)$ or less, there was a significantly greater risk of pulmonary hypertension. Neutrophil volume $\leq 141$ indicated gastrointestinal tract involvement.
\end{abstract}

Conclusions: Simple hematological parameters can be used for predicting SSc-related organ involvements.

Keywords: systemic sclerosis, hematologic parameters, capillaroscopy

Received: 4 May 2020 | Returned for modification: 18 June 2020 | Accepted: 1 July 2020

\section{Introduction}

Systemic sclerosis (SSc) is an autoimmune disease characterized by obliterative vasculopathy and fibrosis of the skin and visceral organs, and it has a high mortality rate compared to other rheumatic diseases. In light of epidemiologic studies conducted between 1969 and 2006, it was reported that SSc showed geographic variations, its prevalence was 7 to 489 per million, and the incidence was 0.6 to 122 per million/year. SSc is more common in women than in men, as is true for most autoimmune rheumatic diseases. Its incidence peaks in the 5 th decade (1). The pathogenesis of SSc is complex and not fully understood (2).

SSc exhibits great heterogeneity in both its clinical and serologic characteristics. SSc is mainly based on the presence of characteristic clinical findings and is supported by specific serologic abnormalities. Antinuclear antibodies (ANA) are present in approximately 90 to $95 \%$ of patients with SSc. In 2013, the American College of Rheumatology / European League against Rheumatism (ACR/EULAR) issued new criteria that had 91\% sensitivity and $92 \%$ specificity for classification of SSc (3). It is well known that the clinical manifestations of SSc based on skin involvement range from limited cutaneous SSc (lcSSc), which indicates a better prognosis, to diffuse cutaneous SSc (dcSSc), in which more severe complications develop (3, 4). Extracutaneous manifestations of SSc include the gastrointestinal tract (GIT) (90\%); musculoskeletal system (45-90\%), cardiac (23-32\%), and renal involvements $(0.5-10 \%)$; progressive interstitial lung disease (ILD) (25-30\%); and pulmonary hypertension (PAH) (15\%), each with various clinical presentations (5-8).

In an international collaborative meta-analysis, it was found that SSc corresponded to a 1.5- to 7.2-times greater mortality risk compared to the general population, and the presence of antitopoisomerase I antibody (Anti-Scl-70) and renal, cardiac, and pulmonary involvement increased the risk of death by 1.3, 1.9, 2.8, and 1.6 times, respectively (9). Pulmonary involvement is the most important cause of morbidity and mortality in the course of SSc (10). Prediction of the course of disease may cause a difference in the choice of treatment because early, targeted, and intensive therapy is key to success in SSc (11); however, the prognosis is difficult to predict in many cases. The interpretation of serological results is often used as a conventional method for predicting the course of disease $(11,12)$.

Complete blood count (CBC) parameters and neutrophil/ lymphocyte (NLR), monocyte/lymphocyte (MLR), and platelet/ lymphocyte (PLR) ratios, which are considered biomarkers of systemic inflammation, have been reported many times in cardiovascular diseases and various cancers, as well as in many rheumatological diseases such as systemic lupus erythematosus (SLE), rheumatoid arthritis (RA), and SSc (13-17).

Studies related to the usefulness of globally available and inexpensive CBC tests to assess the severity of SSc are still lacking. This study investigated the relationship between clinical features, autoantibodies, and especially CBC parameters with various organ involvements.

\section{Method}

\section{Study population and design}

Patients with SSc were recruited from the Department of Rheuma 
tology between January 2016 and June 2019, provided that the were in their first presentation of their disease. Adults that gave written informed consent (130 patients and 129 healthy controls) were enrolled in the study. Data were obtained from the electronic registration database. To be included in the study, any medication (iron, immunosuppressive/immunomodulatory drugs, vitamin supplements, etc.) that might affect hematological parameters could not have been prescribed in the previous 3 months. For this purpose, the drug records of all individuals included in the study were evaluated through the Social Security Institution's Medula system. Patients with cancer, severe infection, chronic hematologic disease, tuberculosis, or primary or secondary (except SSc) heart and lung disease were excluded. Healthy controls were randomly selected from patients without any known disease admitted to the checkup outpatient clinic.

This single-center cross-sectional study was approved by the ethics committee (decision no. 2019/276) and was performed according to the principles of the Declaration of Helsinki.

\section{Classification criteria}

We accepted patients that satisfied the 2013 ACR/EULAR criteria as SSc. The patients were grouped as lcSSc and dcSSc, based on the classification system proposed by LeRoy et al. (8). Organ involvement was evaluated according to clinical symptoms and results of various diagnostic tests. The musculoskeletal disease was defined as arthritis, joint contractures, and myositis based on radiographic and laboratory data. Although the esophagus is the most frequently affected part of the GIT in SSc, it can affect any part of the tract, from the oral cavity to the large intestine, as well as the liver and pancreas $(7,8)$. GIT involvement was defined as the presence of clinical symptoms such as dysphagia, reflux, gastritis, dyspepsia, and diarrhea. The GIT involvement of the patients was decided by combining clinical data with laboratory, endoscopy, and barium X-ray results. The diagnostic criteria for ILD were based on the presence of limited/diffuse ground-glass or honeycomb opacity on high-resolution computed tomography (HRCT). PAH was accepted if pulmonary capillary wedge pressure (PCWP) < $15 \mathrm{~mm} \mathrm{Hg}$ and mean pulmonary artery pressure (PAP) > $25 \mathrm{~mm} \mathrm{Hg}$ in right heart catheterization after measuring systolic $\mathrm{PAP} \geq 40 \mathrm{~mm} \mathrm{Hg}$ on Doppler echocardiography. Findings of nailfold capillaroscopy (NC) were accepted as the presence of neoangiogenesis, capillary elongation/tortuosity, reduced capillary density, avascular area, hemorrhage, giant loops, and abnormal blood flow.

\section{Laboratory measurements}

Blood was analyzed in ethylenediaminetetraacetic acid (EDTA) tubes to obtain CBC results, including the platelet, lymphocyte, neutrophil, and monocyte counts, red cell distribution width (RDW), mean platelet volume (MPV), and lymphocyte, neutrophil, monocyte, and eosinophil volumes for each subject using an automatic blood counting system (Beckman Coulter LH 780, Brea, California, USA). NLR, MLR, and PLR were obtained using the ratio of neutrophil, monocyte, and platelet counts to lymphocyte counts, respectively. The erythrocyte sedimentation rate (ESR; o-20 mm/hour) and C-reactive protein (CRP; $\mathrm{O}-8 \mathrm{mg} / \mathrm{l}$ ) of the patient and control groups and also autoantibodies (ANA titer; antiScl-70; anti-centromere, ACA; anti-PM/Scl; anti-RNP; anti-Ro52; and rheumatoid factor, $\mathrm{RF}$ ) of the patient group were recorded.

\section{Statistical analysis}

All procedures were conducted using IBM SPSS Statistics 21.0 and MS-Excel 2010 software. The results are presented as median (interquartile range). Pearson's chi-square and Fisher's exact test were used for categorical variables where appropriate. After checking the normality distribution of scale variables using the Shapiro-Wilk test, independent samples were compared with appropriate significance tests (e.g., the Mann-Whitney $U$ test, Kruskal-Wallis $H$ test, and Spearman rank correlation coefficient). The associations between ESR, CRP, and CBC parameters were examined using Spearman's partial correlation analysis using syntax after controlling for age. Receiver operating characteristic (ROC) analysis was performed to assess the best cutoff value for predicting organ involvement. We also performed a univariate logistic regression for various organ involvements (dependent), establishing predicting factors such as CBC parameters, autoantibodies, clinical features (independent variables), and their odds ratios (OR). Two-sided $p$-values of $<0.05$ were considered statistically significant. Bonferroni correction was applied as post-hoc after the Kruskal-Wallis $H$ test.

\section{Results}

There were no significant differences between the SSc and control groups in terms of mean age ( $52 \pm 15$ vs. $52 \pm 3$ years, respectively) or sex (123 females, 7 males vs. 121 females, 8 males, respectively). More than half $(52.7 \%)$ of the patients had lcSSc and $47.3 \%$ had dcSSc.

ANA $(n=125,96.2 \%)$, anti-Scl7o $(n=49,37.7 \%)$, ACA $(n=43$, $33.1 \%)$, anti-Ro52 $(n=17,13.2 \%), \operatorname{RF}(n=12,9.3 \%)$, anti-PM/Scl $(n=8,6.2 \%)$, and anti-RNP $(n=6,4.7 \%)$ positivities were detected, in order of frequency. Renal failure was not detected in any patients, and the most common organ involvements were GIT (63.1\%), ILD (48.8\%), digital ulcer (20.8\%), and joint involvement (15.4\%). PAH was detected in $13.2 \%$ of patients. When the presence or absence of organ involvement (dcSSc, ILD, PAH) and control groups were compared separately, all groups were identical in terms of age and sex distribution; only the SSc-ILD group was significantly older than the no-ILD and control groups ( $p=0.004$; Table 1). The CBC parameters, PLRs, NLRs, and MLRs in relation to organ involvement (skin, lung, and PAH) and comparison with the control groups are shown in Tables 1 and 2.

There was a positive moderate correlation between ESR and CRP after adjustment for age in the SSc group $(r=0.535, p<0.001)$. Spearman's partial correlation analysis revealed that hemoglobin (Hb; $r=-0.454, p<0.001)$ RDW $(r=0.413, p<0.001)$ levels, and monocyte volume $(r=0.220, p=0.018)$ showed statistically significant correlations with ESR after accounting for age. In addition, $\mathrm{Hb}(r=-0.224, p=0.016)$, RDW $(r=0.333, p<0.001)$ levels, and monocyte volume $(r=0.235, p=0.011)$ showed statistically significant correlations with CRP. NLR, MLR, PLR, and other CBC parameters were not significantly correlated with ESR and CRP $(p>0.05)$. RDW, ESR, and CRP were significantly different in patients with lcSSc and dcSSc $(p<0.05)$.

Lymphocyte, NLR, PLR, ESR, and CRP levels were significantly different in SSc-PAH than in patients without PAH $(p<0.05)$. Lymphocyte, MPV, RDW, ESR, and CRP levels were significantly different in SSc-ILD compared to patients without ILD $(p<0.05)$. The correlations of CBC parameters, NLR, MLR, and PLR with age are also shown in Table 1 because the age distribution was not iden- 
tical. RDW, ESR, CRP, MLR, and lymphocyte volume were found to be significantly correlated with age; RDW, ESR, and CRP were not included in the ROC analysis in SSc-ILD compared to patients without ILD. The ROC analyses of the skin, lung involvement, and pulmonary hypertension are shown in Figure 1 and Table 3 with the area under the ROC curve (AUC) value, cutoff value, $p$-value, sensitivity, and specificity.

Finally, logistic regression analyses were performed to identify the association of various factors with $p$-values $<0.05$ such as age, CBC parameters, NLR, MLR, PLR, ESR, CRP, autoantibodies, and clinical features (other organ involvement, Raynaud's, and dyspnea), and findings (NC) with the risk of SSc-associated assorted organ involvements. ROC analysis was used to determine the best cutoff value for all continuous variables producing significant results in univariate analysis. Accordingly, after being adjusted for age and sex, the odds ratios and significance levels of univariate analyses are shown as in Table 4.

\section{Skin involvement}

Although there was a remarkable risk increase between dcSSc and ANA titer $(>1 / 320)$, this difference was not significant in the univariate analysis (OR $=2.36,95 \% \mathrm{CI}$ : [0.96-5.89]; $p=0.06)$. The presence of anti-Scl-70 (OR $=2.34,95 \% \mathrm{CI}:[1.13-4.85] ; p=0.023)$ and the absence of ACA (OR $=2.92,95 \%$ CI: $[1.34-6.34] ; p=0.007)$ were associated with a greater risk of dcSSc. There was no rela-

Table 1 | Relationship between simple blood tests and lung involvement in patients with systemic sclerosis (SSc) and correlation of parameters with age.

\begin{tabular}{|c|c|c|c|c|c|c|}
\hline & \multirow{2}{*}{$\begin{array}{c}\text { Control } \\
(n=129)\end{array}$} & \multirow{2}{*}{$\begin{array}{c}\text { No-ILD } \\
(n=66)\end{array}$} & \multirow{2}{*}{$\begin{array}{l}\text { SSc-ILD } \\
(n=63)\end{array}$} & \multirow{2}{*}{$p$-value } & \multicolumn{2}{|c|}{ Correlation with age } \\
\hline & & & & & $r$ & $p$-value \\
\hline Age (years) & $51(4)$ & $48(26)$ & $56(20)$ & $0.004^{\mathrm{b}, \mathrm{c}}$ & 1.00 & - \\
\hline Sex (male/female) & $8 / 121$ & $4 / 62$ & $3 / 60$ & 0.918 & - & - \\
\hline Hemoglobin (g/dl) & $13.3(1.6)$ & $13.3(2.0)$ & $12.6(1.7)$ & $0.001^{b, c}$ & -0.04 & 0.514 \\
\hline Neutrophils $\left(10^{9} / \mathrm{l}\right)$ & $3.70(1.45)$ & $4.52(2.42)$ & $4.16(2.26)$ & $0.028^{\mathrm{a}}$ & -0.01 & 0.885 \\
\hline Lymphocytes $\left(10^{9} / \mathrm{l}\right)$ & $2.28(0.94)$ & $2.07(0.74)$ & $1.60(1.09)$ & $<0.001^{\mathrm{a}, \mathrm{b}, \mathrm{c}}$ & -0.08 & 0.392 \\
\hline Monocytes $\left(10^{9} / \mathrm{l}\right)$ & $0.45(0.16)$ & $0.52(0.22)$ & $0.55(0.30)$ & $0.012^{a}$ & 0.07 & 0.418 \\
\hline Platelets $\left(10^{9} / \mathrm{l}\right)$ & 275 (79) & $245(82)$ & 282 (119) & 0.079 & 0.06 & 0.418 \\
\hline MPW (fl) & $8.3(1.1)$ & $8.5(1.2)$ & $8.0(1.4)$ & $0.026^{c}$ & -0.05 & 0.573 \\
\hline RDW (\%) & $13.8(1.2)$ & $14.1(3.1)$ & $15.6(2.3)$ & $<0.001^{\mathrm{a}, \mathrm{b}, \mathrm{c}}$ & 0.22 & 0.012 \\
\hline NLR & $1.59(0.49)$ & $2.06(1.22)$ & $2.31(1.85)$ & $<0.001^{\mathrm{a}, \mathrm{b}}$ & 0.07 & 0.434 \\
\hline MLR & $0.19(0.07)$ & $0.27(0.12)$ & $0.32(0.21)$ & $<0.001^{\mathrm{a}, \mathrm{b}}$ & 0.18 & 0.039 \\
\hline PLR & 113.9 (49.9) & $125.6(82.3)$ & $147.0(111.6)$ & $<0.001^{b}$ & 0.11 & 0.197 \\
\hline Neutrophil volume & $138.8(9.9)$ & $139.9(9.2)$ & $139.9(7.7)$ & 0.945 & 0.10 & 0.286 \\
\hline Lymphocyte volume & $82.8(5.1)$ & $82.1(5.9)$ & $84.0(5.9)$ & 0.072 & 0.23 & 0.012 \\
\hline Monocyte volume & 159.7 (8.9) & $161.2(13.1)$ & $161.9(10.7)$ & 0.703 & 0.12 & 0.202 \\
\hline Eosinophil volume & $151.3(12.2)$ & $151.6(11.7)$ & $149.4(9.3)$ & 0.105 & 0.01 & 0.896 \\
\hline ESR (mm/hour) & $13(12)$ & $13(14)$ & $24(26)$ & $<0.001^{b, c}$ & 0.19 & 0.027 \\
\hline $\mathrm{CRP}(\mathrm{mg} / \mathrm{l})$ & $3.4(2.1)$ & $3.28(4.89)$ & $4.00(5.86)$ & $0.017^{c}$ & 0.28 & 0.001 \\
\hline
\end{tabular}

$\mathrm{MPV}=$ mean platelet volume, $\mathrm{RDW}=$ red cell distribution width, $\mathrm{PLR}=$ platelet/lymphocyte ratio, $\mathrm{NLR}=$ neutrophil/lymphocyte ratio, $\mathrm{MLR}=\mathrm{monocyte} /$ lymphocyte ratio, $\mathrm{ESR}=$ erythrocyte sedimentation rate, $\mathrm{CRP}=\mathrm{C}$-reactive protein, $\mathrm{SSC}$-ILD = systemic sclerosis-associated interstitial lung disease.

a: $p<0.05$ for the difference between the control group and No-ILD group (Kruskal-Wallis $H$ with the Mann-Whitney $U$ test);

b: $p<0.05$ for the difference between the control group and SSc-ILD group (Kruskal-Wallis $H$ with the Mann-Whitney $U$ test);

c: $p<0.05$ for the difference between the No-ILD group and SSc-ILD group (Kruskal-Wallis $H$ with the Mann-Whitney $U$ test).

Data are expressed as median (interquartile range). The Kruskal-Wallis $H$, Mann-Whitney $U$, chi-square tests and Spearman's rho correlation analysis were used. Bonferroni correction was applied as post-hoc after the Kruskal-Wallis $H$ test. Significant values are shown in bold.

Table 2 | Relationship between simple blood tests and skin involvement and pulmonary hypertension (PAH) in patients with systemic sclerosis (SSc).

\begin{tabular}{|c|c|c|c|c|c|c|c|}
\hline & $\begin{array}{c}\text { Control } \\
(n=129)\end{array}$ & $\begin{array}{c}\text { IcSSc } \\
(n=68)\end{array}$ & $\begin{array}{c}\text { dcSSc } \\
(n=61)\end{array}$ & $p$-value & $\begin{array}{c}\text { No-PAH } \\
(n=112)\end{array}$ & $\begin{array}{c}\text { SSc-PAH } \\
(n=17)\end{array}$ & $p$-value \\
\hline Age (years) & $51(4)$ & $49(23)$ & $45(22)$ & 0.074 & $51(22)$ & $58(18)$ & 0.057 \\
\hline Sex (male/female) & $8 / 121$ & $4 / 64$ & $3 / 58$ & 0.939 & $6 / 106$ & $1 / 16$ & 0.835 \\
\hline Hemoglobin (g/dl) & $13.3(1.6)$ & $13.1(1.9)$ & $12.8(2.2)$ & $0.017^{b}$ & $13.0(1.8)$ & $12.2(2.0)$ & $0.015^{b}$ \\
\hline Neutrophils $\left(10^{9} / \mathrm{l}\right)$ & $3.70(1.45)$ & $4.30(2.42)$ & $4.01(2.26)$ & $0.021^{a}$ & $4.23(2.42)$ & $4.13(2.04)$ & $0.021^{a}$ \\
\hline Lymphocytes $\left(10^{9} / \mathrm{l}\right)$ & $2.28(0.94)$ & $1.94(0.87)$ & $1.79(1.18)$ & $<0.001^{a, b}$ & $1.91(0.99)$ & $1.31(1.03)$ & $<0.001^{a, b, c}$ \\
\hline Monocytes $\left(10^{9} / \mathrm{l}\right)$ & $0.45(0.16)$ & $0.52(0.28)$ & $0.54(0.27)$ & $0.013^{a}$ & $0.53(0.24)$ & $0.41(0.40)$ & $0.005^{a}$ \\
\hline Platelets $\left(10^{9} / \mathrm{l}\right)$ & 275 (79) & $257(106)$ & $271(107)$ & 0.599 & $270(113)$ & 249 (147) & $0.042^{b}$ \\
\hline MPW (fl) & $8.3(1.1)$ & $8.5(1.4)$ & $8.1(1.3)$ & 0.177 & $8.3(1.5)$ & $8.6(1.3)$ & 0.863 \\
\hline RDW (\%) & $13.8(1.2)$ & $14.3(2.9)$ & $15.6(2.3)$ & $<0.001^{a, b, c}$ & $14.9(2.7)$ & $15.8(2.2)$ & $<0.001^{\mathrm{a}, \mathrm{b}}$ \\
\hline NLR & $1.59(0.49)$ & $2.09(1.70)$ & $2.20(1.65)$ & $<0.001^{a, b}$ & $2.10(0.63)$ & $3.38(3.78)$ & $<0.001^{a, b, c}$ \\
\hline MLR & $0.19(0.07)$ & $0.27(0.14)$ & $0.31(0.17)$ & $<0.001^{\mathrm{a}, \mathrm{b}}$ & $0.29(0.15)$ & $0.40(0.30)$ & $<0.001^{\mathrm{a}, \mathrm{b}}$ \\
\hline PLR & $113.9(49.9)$ & $142.6(86.9)$ & $144.2(101.3)$ & $0.002^{a, b}$ & $141.0(90.7)$ & $147.5(106.3)$ & $0.001^{a, c}$ \\
\hline Neutrophil volume & $138.8(9.9)$ & $141.0(12.4)$ & $139.9(7.3)$ & 0.550 & $139.3(9.5)$ & $141.4(3.4)$ & 0.726 \\
\hline Lymphocyte volume & $82.8(5.1)$ & $83.4(6.6)$ & $83.0(6.3)$ & 0.720 & $83.3(7.0)$ & $84.0(5.2)$ & 0.635 \\
\hline Monocyte volume & $159.7(8.9)$ & $162.0(12.8)$ & $161.3(8.7)$ & 0.983 & $160.9(11.6)$ & $164.6(9.2)$ & 0.193 \\
\hline Eosinophil volume & $151.3(12.2)$ & $151.6(11.2)$ & $149.4(10.4)$ & 0.084 & $149.6(11.2)$ & $150.4(9.0)$ & 0.114 \\
\hline ESR (mm/hour) & $13(12)$ & $14(14)$ & $22(20)$ & $0.009^{b, c}$ & $15(18)$ & $29(30)$ & $0.003^{b, c}$ \\
\hline $\mathrm{CRP}(\mathrm{mg} / \mathrm{l})$ & $3.4(2.1)$ & $3.3(3.6)$ & $4.0(6.0)$ & $0.033^{c}$ & $3.4(4.3)$ & $7.1(6.4)$ & $0.017^{\mathrm{b}, \mathrm{c}}$ \\
\hline
\end{tabular}

$\mathrm{MPV}=$ mean platelet volume, $\mathrm{RDW}=$ red cell distribution width, $\mathrm{PLR}=$ platelet/lymphocyte ratio, NLR = neutrophil/lymphocyte ratio, $M L R=\mathrm{monocyte} /$ lymphocyte ratio, $\mathrm{ESR}=$ erythrocyte sedimentation rate, $\mathrm{CRP}=\mathrm{C}$-reactive protein, lcSSc = limited cutaneous systemic sclerosis, dcSSc $=$ diffused cutaneous systemic sclerosis, $\mathrm{SSc}-\mathrm{PAH}=$ systemic sclerosis-associated pulmonary hypertension.

a: $p<0.05$ for the difference between the control group and IcSSc/No-PAH group (Kruskal-Wallis $H$ with the Mann-Whitney $U$ test);

$\mathrm{b}: p<0.05$ for the difference between the control group and dcSSc/SSc-PAH group (Kruskal-Wallis $H$ with the Mann-Whitney $U$ test);

c: $p<0.05$ for the difference between the IcSSc/No-PAH group and dcSSc/SSc-PAH group (Kruskal-Wallis $H$ with the Mann-Whitney $U$ test).

The Kruskal-Wallis $H$, Mann-Whitney $U$, and chi-square tests were used. Bonferroni correction was applied as post-hoc after the Kruskal-Wallis $H$ test. Significant values are shown in bold. 
tionship between other autoantibodies (e.g., RF, RO52, $\mathrm{PM} / \mathrm{Scl}$, RNP) and skin involvement ( $p>0.05)$. Elevation of ESR $(>20 \mathrm{~mm} /$ hour) significantly increased the risk of dcSSc development alone $(\mathrm{OR}=2.16,95 \% \mathrm{CI}:[1.06-4.41] ; p=0.035)$.

\section{GIT and joint involvements}

About $70 \%$ of patients with GIT involvement had esophageal involvement, whereas no liver and pancreas involvement were detected. There was no relationship between skin and GIT involvement. GIT involvement was found to be significantly greater in patients with SSc-ILD (OR = 2.40, 95\% CI: [1.15-5.00]; $p=0.020$ ). In addition, neutrophil volume $\leq 141.0$ indicated GIT involvement $(\mathrm{OR}=2.46,95 \% \mathrm{CI}:[1.13-5.35] ; p=0.023)$.

The risk of joint symptoms was greater in patients without ILD $(\mathrm{OR}=3.12$, 95\% CI: [1.05-9.27]; $p=0.040)$.

\section{Interstitial lung disease}

It was noteworthy that dyspnea was the most crucial factor, with a 390-fold greater risk for SSc-ILD (OR = 390.0, 95\% CI: [47.8-3176.1]; $p<0.001$ ). Dyspnea was present in $85.7 \%$ of SSc-ILD; all patients with dyspnea-free ILD had limited ground-glass subtype ILD. Sub- sequently, the risk of ILD was 6.52 times greater in generalized skin involvement ( $\mathrm{OR}=6.52,95 \% \mathrm{CI}$ : [3.01-14.17]; $p<0.001)$. In contrast to ANA titers, the absence of ACA was associated with a greater risk of ILD (OR $=5.3,95 \%$ CI: [2.31-12.16]; $p \leq 0.001)$. Age (> 50 years), ESR ( $>25 \mathrm{~mm} /$ hour), $\mathrm{Hb}(\leq 13.0 \mathrm{~g} / \mathrm{dl})$, lymphocyte count $\leq 1,900$ $\left(10^{3} / \mathrm{ml}\right)$, and MPV $(\leq 8.0 \mathrm{fl})$ were also evaluated separately, and statistically significant results were obtained to estimate the risk of ILD $(p<0.05)$.

\section{Pulmonary hypertension and digital ulcers}

The presence of anti-RNP antibodies was found to be significant in predicting greater risk of $\mathrm{PAH}$ as the most crucial factor in univariate analysis (OR $=16.77,95 \% \mathrm{CI}$ : [2.79-100.66]; $p=0.002)$. The risk of PAH was 5.78 times greater in patients with dcSSc $(\mathrm{OR}=$ 5.78, 95\% CI: [1.56-21.41]; $p=0.009$ ). It was determined that the presence of dyspnea indicating ILD did not correspond to a significantly greater risk for PAH (OR $=2.73,95 \% \mathrm{CI}$ : [0.94-7.91]; $p$ $=0.064)$. We found that patients with abnormal NC had a greater risk of $\mathrm{PAH}(\mathrm{OR}=3.09,95 \% \mathrm{CI}$ : $[1.08-8.85] ; p=0.035)$. When the lymphocyte count was $1,400\left(10^{3} / \mathrm{ml}\right)$ or less, there was a marked and significant increase in the risk of $\mathrm{PAH}(\mathrm{OR}=6.72,95 \% \mathrm{CI}$ : [2.25-20.04]; $p=0.001)$.
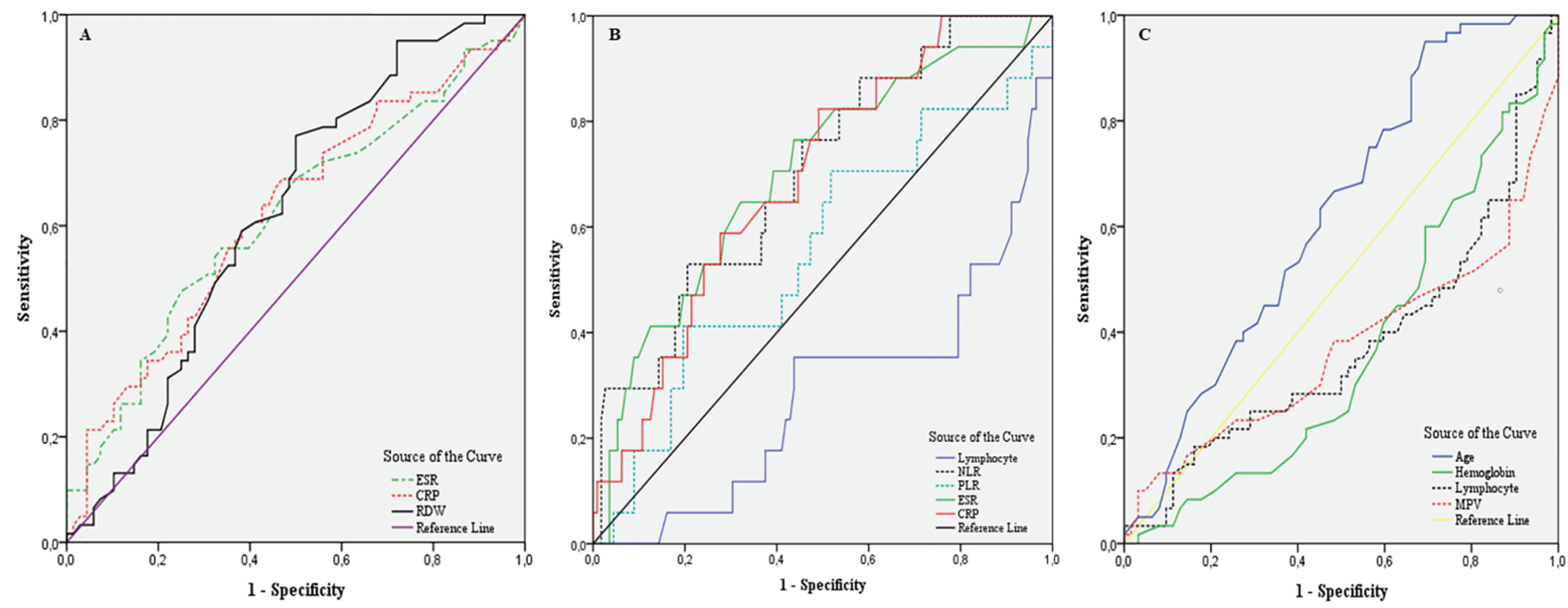

Figure 1 | Receiver operating characteristic curve (ROC) analysis for distinguishing between organ involvement associated with systemic sclerosis (SSc): a) limited SSc vs. diffuse cutaneous SSc; b) absence vs. presence of SSc-associated pulmonary hypertension; c) absence vs. presence of SSc-associated interstitial lung disease.

Table 3 | Receiver operating characteristic (ROC) curve analysis to distinguish organ involvements.

\begin{tabular}{|c|c|c|c|c|c|}
\hline & AUROC & Cut-off value & Sensitivity (\%) & Specificity (\%) & $p$-value \\
\hline \multicolumn{6}{|l|}{ IcSSc vs. dcSSc } \\
\hline ESR (mm/hour) & 0.617 & 19.50 & 54.1 & 67.6 & 0.022 \\
\hline $\mathrm{CRP}(\mathrm{mg} / \mathrm{l})$ & 0.622 & 4.5 & 55.7 & 50.8 & 0.017 \\
\hline RDW (\%) & 0.623 & 14.2 & 77.0 & 50.0 & 0.016 \\
\hline \multicolumn{6}{|l|}{ No-PAH vs. SSc-PAH } \\
\hline Lymphocytes $\left(10^{9} / \mathrm{l}\right)$ & 0.714 & 1.395 & 64.7 & 79.5 & 0.005 \\
\hline NLR & 0.703 & 2.209 & 76.5 & 54.5 & 0.007 \\
\hline PLR & 0.554 & - & - & - & 0.473 \\
\hline ESR (mm/hour) & 0.701 & 19.50 & 70.6 & 60.7 & 0.008 \\
\hline $\mathrm{CRP}(\mathrm{mg} / \mathrm{l})$ & 0.692 & 6.09 & 58.8 & 72.3 & 0.011 \\
\hline \multicolumn{6}{|l|}{ No-ILD vs. SSc-ILD } \\
\hline Age (years) & 0.649 & 49.5 & 65.1 & 56.1 & 0.004 \\
\hline Hemoglobin (g/dl) & 0.634 & 12.85 & 45.0 & 37.1 & 0.011 \\
\hline Lymphocytes $\left(10^{9} / \mathrm{l}\right)$ & 0.626 & 1.885 & 44.3 & 39.4 & 0.014 \\
\hline MPV (fl) & 0.632 & 8.0 & 60.0 & 66.6 & 0.010 \\
\hline
\end{tabular}

IcSSc = limited cutaneous systemic sclerosis, dcSSc = diffused cutaneous systemic sclerosis, SSc-ILD = systemic sclerosis-associated interstitial lung disease, $\mathrm{SSC}-\mathrm{PAH}=$ systemic sclerosis-associated pulmonary hypertension, AUROC $=$ area under the receiver operating characteristic curve, $\mathrm{MPV}=$ mean platelet volume, $\mathrm{RDW}=$ red cell distribution width, PLR = platelet/lymphocyte ratio, NLR = neutrophil/lymphocyte ratio, ESR = erythrocyte sedimentation rate, CRP = C-reactive protein.

Significant values are shown in bold. 
Table 4 | Evaluation of factors associated with organ involvement by univariate logistic regression analysis.

\begin{tabular}{|c|c|c|c|c|c|c|}
\hline \multirow{2}{*}{ Univariate logistic regression analysis } & \multirow{2}{*}{$\beta \mathbf{i}$} & \multirow{2}{*}{ Odds ratio } & \multicolumn{2}{|c|}{$95 \% \mathrm{Cl}$} & \multirow{2}{*}{ Wald value } & \multirow{2}{*}{$p$-value } \\
\hline & & & Lower & Upper & & \\
\hline \multicolumn{7}{|l|}{ Generalized skin involvement $(n=61 / 129)$} \\
\hline Age $>50$ years & 0.61 & 2.84 & 0.91 & 3.71 & 2.91 & 0.088 \\
\hline $\mathrm{ESR}>20 \mathrm{~mm} /$ hour & 0.77 & 2.16 & 1.06 & 4.41 & 4.47 & 0.035 \\
\hline $\mathrm{CRP}>4.5 \mathrm{mg} / \mathrm{l}$ & 0.71 & 2.02 & 0.99 & 4.13 & 3.74 & 0.053 \\
\hline RDW (\%) & 0.13 & 1.13 & 0.97 & 1.33 & 2.42 & 0.119 \\
\hline ANA > $1 / 320(+)$ & 0.87 & 2.38 & 0.96 & 5.89 & 3.54 & 0.060 \\
\hline Scl-70 (+) & 0.85 & 2.34 & 1.13 & 4.85 & 5.19 & 0.023 \\
\hline $\mathrm{ACA}(-)$ & 1.07 & 2.92 & 1.34 & 6.34 & 7.27 & 0.007 \\
\hline \multicolumn{7}{|l|}{ Digital ulcer $(n=27 / 130)$} \\
\hline Nail fold capillaroscopy (+) & 2.51 & 12.26 & 1.59 & 94.23 & 5.80 & 0.016 \\
\hline \multicolumn{7}{|l|}{ GIT involvement $(n=74 / 116)$} \\
\hline Neutrophil volume $\leq 141.0$ & 0.90 & 2.46 & 1.13 & 5.35 & 5.18 & 0.023 \\
\hline Interstitial lung disease & 0.87 & 2.40 & 1.15 & 5.00 & 5.40 & 0.020 \\
\hline \multicolumn{7}{|l|}{ Joint involvement $(n=20 / 130)$} \\
\hline No interstitial lung disease & 1.14 & 3.12 & 1.05 & 9.27 & 4.21 & 0.040 \\
\hline \multicolumn{7}{|c|}{ Interstitial lung disease in all SSc patients $(n=63 / 129)$} \\
\hline Age $>50$ years & 0.73 & 2.07 & 1.03 & 4.19 & 4.13 & 0.042 \\
\hline Generalized skin involvement & 1.88 & 6.52 & 3.01 & 14.17 & 22.67 & $<0.001$ \\
\hline Dyspnea (+) & 5.97 & 390.00 & 47.80 & 3176.10 & 31.08 & $<0.001$ \\
\hline Hemoglobin $\leq 13.0 \mathrm{~g} / \mathrm{dl}$ & 0.87 & 2.39 & 1.15 & 4.95 & 5.51 & 0.019 \\
\hline ESR $>25 \mathrm{~mm} /$ hour & 1.28 & 3.60 & 1.62 & 8.00 & 9.88 & 0.002 \\
\hline RDW (\%) & 0.07 & 1.07 & 0.92 & 1.24 & 0.80 & 0.372 \\
\hline Lymphocyte $\leq 1.910^{9} / \mathrm{l}$ & 0.72 & 2.06 & 1.02 & 4.16 & 4.09 & 0.043 \\
\hline$M P V \leq 8.0 \mathrm{fl}$ & 0.79 & 2.20 & 1.08 & 4.48 & 4.72 & 0.030 \\
\hline ACA (-) & 1.67 & 5.30 & 2.31 & 12.16 & 15.50 & $<0.001$ \\
\hline \multicolumn{7}{|l|}{ Pulmonary hypertension $(n=17 / 129)$} \\
\hline Generalized skin involvement & 1.75 & 5.78 & 1.56 & 21.41 & 6.89 & 0.009 \\
\hline Dyspnea (+) & 1.01 & 2.73 & 0.94 & 7.91 & 3.42 & 0.064 \\
\hline Sm/RNP (+) & 2.82 & 16.77 & 2.79 & 100.66 & 9.51 & 0.002 \\
\hline NLR > 2.2 & 1.32 & 3.75 & 1.15 & 12.21 & 4.82 & 0.028 \\
\hline$M L R>0.35$ & 1.32 & 3.73 & 1.31 & 10.68 & 6.04 & 0.014 \\
\hline $\mathrm{ESR}>20 \mathrm{~mm} /$ hour & 1.08 & 2.94 & 1.01 & 8.54 & 3.94 & 0.047 \\
\hline $\mathrm{CRP}>6 \mathrm{mg} / \mathrm{l}$ & 1.31 & 3.73 & 1.31 & 10.68 & 6.04 & 0.014 \\
\hline Lymphocyte $\leq 1.410^{9} / \mathrm{l}$ & 1.91 & 6.72 & 2.25 & 20.04 & 11.69 & 0.001 \\
\hline Nail fold capillaroscopy (+) & 1.13 & 3.09 & 1.08 & 8.85 & 4.43 & 0.035 \\
\hline
\end{tabular}

$\beta \mathrm{i}=$ regression coefficient, $\mathrm{Cl}=$ confidence interval, $\mathrm{MPV}=$ mean platelet volume, $\mathrm{RDW}=$ red cell distribution width, $\mathrm{NLR}=\mathrm{neutrophil} / \mathrm{lymphocyte}$ ratio, $\mathrm{MLR}=$ monocyte/lymphocyte ratio, $\mathrm{ESR}=$ erythrocyte sedimentation rate, $\mathrm{CRP}=\mathrm{C}$-reactive protein, $\mathrm{ANA}=$ antinuclear antibody, $\mathrm{Scl}-70=$ anti-topoisomerase $\mathrm{I}$ antibody, $A C A=$ anti-centromere antibody.

Significant values are shown in bold.

\section{Discussion}

Mortality in SSc is the result of organ involvement, with lung disease (either ILD or PAH) being the most prominent risk factor for death (15). Previous studies reported risk factors for poor survival in SSc such as male sex, diffuse cutaneous subtype, age at disease onset, African origin, presence of anti-Scl-70 antibodies, and specific organ involvement. Identifying autoantibodies is clinically useful in helping diagnosis and in predicting the development of certain clinical manifestations and prognosis (18). ACA is associated with limited cutaneous involvement and $\mathrm{PAH}$, whereas antiScl7o antibodies are associated with diffuse skin involvement and pulmonary fibrosis. It has been known for a long time that ACA is a predictor for a favorable prognosis, and that anti-Scl-70 is associated with a poor prognosis and SSc-related mortality (19). This study confirms these data.

Yang et al. compared the blood cell count ratios of 1,139 patients with systemic autoimmune rheumatic diseases (SARDs). They emphasized that especially NLR and MLR might reflect inflammatory status in SARDs (16). In recent years, accumulating evidence has indicated the potentially great diagnostic and prognostic value of complementary components of $\mathrm{CBC}$, and particularly blood cell count ratios for various chronic inflammatory diseases (e.g., psoriasis, RA, SLE, Behçet disease, SSc, and multiple sclerosis) and cancers (lymphoma and solid cancers) (16,
20-27). It has been reported that MLR, PLR, MPV, and diverse cell counts have a prognostic value in some diseases, although almost all studies have focused on NLR alone (20, 22, 25, 26, 28). In this study, unlike previous studies, we present a comprehensive analysis by considering almost all the variables in CBC parameters as well as immunological, clinical parameters.

In this study, age ( $>50$ years), ESR $(>20 \mathrm{~mm} /$ hour $)$, CRP $(>4.5$ $\mathrm{mg} / \mathrm{l})$, RDW (14.2\%), ANA titer (> 1/320), anti-Scl-70 (+), and ACA $(-)$ parameters were found to be associated with dcSSc. However, in the univariate analysis, only ESR, anti-Scl-70, and ACA were able to produce significant results in the prediction of dcSSc risk. Similar to our study, Zhao et al. found a moderate correlation between CRP and RDW, and they reported no relationship between RDW and dcSSc (29). Conversely, Farkas et al. reported that RDW elevation was associated with dcSSc, as well as cardiopulmonary findings (30). In this study, it was interesting that, although RDW was significantly higher in dcSSc than in the control and lcSSc groups, it did not significantly increase the risk of developing dcSSc.

Current data suggest that esophageal disease may independently contribute to or be a source of ILD in SSc (31). Observational studies indicate that SSc patients with more active reflux disease ultimately develop more advanced ILD. It is not known whether particulate reflux or acid reflux or both are involved in the development of ILD (32). It was determined that the risk of 
GIT involvement was 2.4 times greater in patients with SSc-ILD, and the risk of joint involvement was approximately three times greater in patients without ILD. The relationship between GIT involvement and the decrease in neutrophil volume was quite remarkable. However, neutrophil volume has not been analyzed to date, although NLR, MLR, and MPV have been reported not to be associated with SSc-associated GIT involvement (33).

Although 54 of 63 patients with ILD had dyspnea, only nine were asymptomatic. All patients with dyspnea-free ILD had a limited ground-glass appearance on HRCT. Dyspnea was present in patients with all diffuse ground-glass and limited/diffuse honeycombing ILD. According to our study, in patients with SSc, due to the 390 -fold greater ILD risk in the presence of dyspnea, lung involvement should be considered until proven otherwise. Patients with dcSSc had an approximately 6.5-fold increase in ILD compared to lcSSc. ACA negativity associated with dcSSc also indicated ILD more strongly, with a 5.3-fold greater risk. Parameters such as age (> 50 years), ESR (> $25 \mathrm{~mm} /$ hour), $\mathrm{Hb}(\leq 13.0 \mathrm{~g} / \mathrm{dl})$, lymphocyte count $\left(\leq 1,900 \times 10^{3} / \mathrm{ml}\right)$, and MPV $(\leq 8.0 \mathrm{fl})$ individually pointed to about a 2 - to 2.5 -fold greater SSc-ILD risk.

Jung et al. reported that SSc-ILD could be predicted in patients with SSc with NLRs above 2.59 (70\% sensitivity, 72\% specificity) and that NLR could be used as a marker for SSc-ILD (34). Atilla et al. reported that $\mathrm{Hb}$ and counts of leukocytes and platelets were not different in patients with SSc with or without ILD, whereas NLR was above 3.21, indicating ILD ( $81 \%$ sensitivity, $81 \%$ specificity) (35). In this study, NLR was higher in patients with SSc than in healthy subjects; however, it did not indicate lung involvement. In the two studies mentioned above, unlike this study, only NLR was investigated, and other whole blood count parameters were not evaluated. Yayla et al. evaluated the relationship between SSc-associated organ involvement and NLR, MLR, and MPV, but found that none showed significant differences for SSc-ILD (33).

In estimating SSc-PAH, the most important test was determined to be anti-RNP positivity, with a 16.7-fold greater risk. It was unusual that the risk of SSc-PAH was approximately 5.8 times greater in dcSSc associated with SSc-ILD, though there was no significantly greater risk of SSc-PAH in the presence of dyspnea $(p=0.064)$. Severe NC changes have been reported to increase the risk of SScPAH (36-38). Similarly, it was found that the risk of PAH was about three times greater with abnormalities in NC. Each of ESR $(>20$ $\mathrm{mm} /$ hour), CRP (> $6 \mathrm{mg} / \mathrm{l}), \mathrm{NLR}(>2.2)$, and MLR (> 0.35) increased the risk of PAH approximately three to four times, separately. There was a significant 6.7-fold greater risk of $\mathrm{PAH}$ in patients with SSc with lymphocyte count $\leq 1,400\left(10^{3} / \mathrm{ml}\right)(p=0.001)$.

Özpelit et al. reported that NLR might be useful in assessing the severity of disease in patients with idiopathic PAH (34). In a study of patients with SSc, NLR was reported to be associated with digital ulcers, PAH, and ILD. Yayla et al. confirmed that NLR was associated with SSc-PAH and reported that NLR and MLR were associated with digital ulcers (33). However, we found that no clinical or laboratory parameters other than NC indicated digital ulcers with a significant association (OR $=12.26$, 95\% CI: [1.5994.53]; $p=0.016)$. RDW and MPV are considered a complementary measure of multiple pathologic processes that simultaneously occur in SSc, including oxidative stress, thrombosis, inflammation, and endothelial dysfunction (39-41). Accordingly, many studies have examined the relationship between these parameters and micro-macrovascular events, and provided evidence that there is an association with digital ulcers and/or SSc-PAH $(29,41,43)$. According a study by Zhao et al. (28), RDW elevation (> 14.3\%) was reported to increase the risk of PAH by 3.31 times when combined with anti-U1-RNP, but our study did not support this.

The most important limitation of the study is its retrospective nature. A retrospective bias should never be ignored, although we were careful with patient selection because we predicted the possible effect of drug use on hematological parameters (41). It is noteworthy that almost all similar studies in the literature are retrospective. We believe that contradictory results in the literature result from a retrospective bias. Therefore, there is a need for a prospective controlled study with more subjects for reproducibility. In addition, the weakest point of this article is that the significant results of sensitivity and specificity deduced from the ROC curves are quite low, although reasonable results were obtained from logistic regression analysis.

Determining multicollinearity is an exhaustive process in SPSS Statistics that requires creating any dummy variables that are needed and running multiple linear regression procedures. In the case of so many interrelated data, the technical difficulty of building reliable multiple logistic regression models was considered. We could not apply multiple logistic regression models due to multicollinearity problems. However, according to the univariate analysis, it was possible to obtain an idea about the importance and usefulness of various parameters related to organ involvement by listing different ORs.

\section{Conclusions}

As can be seen above, related publications generally focus on several parameters such as NLR, MPV, and RDW, and they have conflicting results. In this study, $\mathrm{CBC}$ parameters were considered along with a comprehensive analysis with many clinical presentations and various hematological and immunological data from SSc patients. Our study revealed that CBC parameters such as NLR, MLR, MPV, Hb, lymphocyte counts, and neutrophil volume were associated with assorted organ involvements. Although much research has been performed on the subject, we believe that simple hemogram parameters will not be useful enough in daily clinical practice because of their low predictive values. However, this needs to be supported by prospective controlled studies. We believe that our study may have implications for future research and clinical practice in terms of its results.

\section{References}

1. Asano Y. Systemic sclerosis. J Dermatol. 2018;45:128-38.

2. Wielosz E, Majdan M. Clinical and serological diversity in systemic sclerosis. Wiad Lek. 2018;71:78-83.

3. Sierra-Sepúlveda A, Esquinca-González A, Benavides-Suárez SA, Sordo-Lima DE, Caballero-Islas AE, Cabral-Castañeda AR, et al. Systemic sclerosis pathogenesis and emerging therapies, beyond the fibroblast. Biomed Res Int. 2019:4569826.
4. Amoda O, Ravat V, Datta S, Saroha B, Patel RS. Trends in demographics, hospitalization outcomes, comorbidities, and mortality risk among systemic sclerosis patients. Cureus. 2018;10:e2628.

5. Salazar G, Mayes MD. Genetics, epigenetics, and genomics of systemic sclerosis. Rheum Dis Clin North Am. 2015;41:345-66. 
6. Singh D, Parihar AK, Patel S, Srivastava S, Diwan P, Singh MR. Scleroderma: an insight into causes, pathogenesis and treatment strategies. Pathophysiology. 2019;26:103-114.

7. Van Den Hoogen F, Khanna D, Fransen J, Johnson SR, Baron M, Tyndall A, et al. 2013 classification criteria for systemic sclerosis: an American College of Rheumatology / European League against Rheumatism collaborative initiative. Ann Rheum Dis. 2013;65:2737-47.

8. LeRoy E, Black C, Fleischmajer R, Jablonska S, Krieg T, Medsger Jr TA, et al. Scleroderma (systemic sclerosis): classification, subsets and pathogenesis. J Rheumatol. 1988;15:202-5.

9. Denton CP, Khanna DJ. Systemic sclerosis. Lancet. 2017;390:1685-99.

10. Giuggioli D, Manfredi A, Lumetti F, Colaci M, Ferri C. Scleroderma skin ulcers definition, classification and treatment strategies our experience and review of the literature. Autoimmun Rev. 2018;17:155-64.

11. Riemekasten G. Progress in systemic sclerosis-early, targeted and intensive therapy is the key to success. Dtsch Med Wochenschr. 2019;144:189-93.

12. Hung G, Mercurio V, Hsu S, Mathai SC, Shah AA, Mukherjee M. Progress in understanding, diagnosing, and managing cardiac complications of systemic sclerosis. Curr Rheumatol Rep. 2019;21:68.

13. Choi MY, Fritzler MJ. Progress in understanding the diagnostic and pathogenic role of autoantibodies associated with systemic sclerosis. Curr Opin Rheumatol. 2016;28:586.

14. Hamaguchi Y. Autoantibody profiles in systemic sclerosis: predictive value for clinical evaluation and prognosis. J Dermatol. 2010;37:42-53.

15. Volkmann ER, Tashkin DP, Sim M, Li N, Goldmuntz E, Keyes-Elstein L, et al. Short-term progression of interstitial lung disease in systemic sclerosis predicts long-term survival in two independent clinical trial cohorts. Ann Rheum Dis. 2019;78:122-30.

16. Yang Z, Zhang Z, Lin F, Ren Y, Liu D, Zhong R, et al. Comparisons of neutrophilmonocyte-, eosinophil-, and basophil-lymphocyte ratios among various systemic autoimmune rheumatic diseases. APMIS. 2017;125:863-71.

17. Ntelis K, Bogdanos D, Dimitroulas T, Sakkas L, Daoussis D. Platelets in systemic sclerosis: the missing link connecting vasculopathy, autoimmunity, and fibrosis? Curr Rheumatol Rep. 2019;21:15.

18. Jeong S, Yang D, Lee W, Kim GT, Kim HS, Ahn HS, et al. Diagnostic value of screening enzyme immunoassays compared to indirect immunofluorescence for anti-nuclear antibodies in patients with systemic rheumatic diseases: a systematic review and meta-analysis. Semin Arthritis Rheum. 2018;48:334-42.

19. Steen VD, Powell DL, Medsger TA Jr. Clinical correlations and prognosis based on serum autoantibodies in patients with systemic sclerosis. Arthritis Rheum. 1988;31:196-203.

20. Kim DS, Shin D, Lee MS, Kim HJ, Kim DY, Kim SM, et al. Assessments of neutrophil to lymphocyte ratio and platelet to lymphocyte ratio in Korean patients with psoriasis vulgaris and psoriatic arthritis. J Dermatol. 2016;43:305-10.

21. Chen Q, Chen D, Xu X, Liu Y, Yin T, Li D. Platelet/lymphocyte, lymphocyte/monocyte, and neutrophil/lymphocyte ratios as biomarkers in patients with rheumatoid arthritis and rheumatoid arthritis-associated interstitial lung disease. Med Sci Monit. 2019;25:6474.

22. Qin B, Ma N, Tang Q, Wei T, Yang M, Fu H, et al. Neutrophil to lymphocyte ratio (NLR) and platelet to lymphocyte ratio (PLR) were useful markers in assessment of inflammatory response and disease activity in SLE patients. Mod Rheumatol. 2016;26:372-6.

23. Rifaioglu EN, Bülbül BŞ, Ekiz Ö, Cigdem AC, Pannonica, et Adriatica. Neutrophil to lymphocyte ratio in Behçet's disease as a marker of disease activity. Acta Dermatovenerol Alp Pannonica Adriat. 2014;23:65-7.

24. Demirci S, Demirci S, Kutluhan S, Koyuncuoglu HR, Yurekli VA. The clinical significance of the neutrophil-to-lymphocyte ratio in multiple sclerosis. Int J Neurosci. 2016;126:700-6.
25. Romano A, Parrinello NL, Vetro C, Chiarenza A, Cerchione C, Ippolito M, et al. Prognostic meaning of neutrophil to lymphocyte ratio (NLR) and lymphocyte to monocyte ration (LMR) in newly diagnosed Hodgkin lymphoma patients treated upfront with a PET-2 based strategy. Ann Hematol. 2018;97:1009-18.

26. Ying HQ, Deng QW, He BS, Pan YQ, Wang F, Sun HL, et al. The prognostic value of preoperative NLR, d-NLR, PLR and LMR for predicting clinical outcome in surgical colorectal cancer patients. Med Oncol. 2014;31:305.

27. Mirsaeidi M, Barletta P, Glassberg MK. Systemic sclerosis associated interstitial lung disease: new directions in disease management. Front Med (Lausanne). 2019;6:248.

28. Abdel Galil SM, Edrees AM, Ajeeb AK, Aldoobi GS, El-Boshy M, Hussain WJP. Prognostic significance of platelet count in SLE patients. Platelets. 2017:28:203-7.

29. Zhao J, Mo H, Guo X, Wang Q, Xu D, Hou Y, et al. Red blood cell distribution width as a related factor of pulmonary arterial hypertension in patients with systemic sclerosis. Clin Rheumatol. 2018;37:979-85.

30. Farkas N, Szabó A, Lóránd V, Sarlós DP, Minier T, Prohászka Z, et al. Clinical usefulness of measuring red blood cell distribution width in patients with systemic sclerosis. Rheumatology (Oxford). 2014;53:1439-45.

31. Richardson C, Agrawal R, Lee J, Almagor O, Nelson R, et al. Esophageal dilatation and interstitial lung disease in systemic sclerosis: a cross-sectional study. Semin Arthritis Rheum. 2016;46:109-14.

32. McFarlane IM, Bhamra MS, Kreps A, Iqbal S, Al-Ani F, Saladini-Aponte C, et al. Gastrointestinal manifestations of systemic sclerosis. Rheumatology (Sunnyvale). 2018;8:235

33. Yayla M, İlgen U, Okatan I, Yurteri EU, Torğutalp M, Dinçer AK, et al. Association of haematological parameters with disease manifestations, activity, and severity in patients with systemic sclerosis. Clin Rheumatol. 2020;39:77-83.

34. Jung JH, Lee YM, Lee EG, Yoo WH, Lee WS. Neutrophil-to-lymphocyte ratio in diagnosis of systemic sclerosis for prediction of interstitial lung disease. J Rheum Dis. 2017;24:138-42.

35. Atilla N, Cetin GY, Balkarli A. Association of neutrophil/lymphocyte ratio with the degree of interstitial lung disease in systemic sclerosis. Turk J Med Sci. 2016;46: 1871-4.

36. Riccieri V, Vasile M, Iannace N, Stefanantoni K, Sciarra I, Vizza CD, et al. Systemic sclerosis patients with and without pulmonary arterial hypertension: a nailfold capillaroscopy study. Rheumatology (Oxford). 2013;52:1525-8.

37. Groseanu L, Paraschiva P, Balanescu A, Bojinca V, Belinski DO, Borangiu A, et al. The role of nailfold capillaroscopy in monitoring lung involvement in systemic sclerosis. Ro J Rheumatol. 2019;28:17-22.

38. Kubo S, Smith V, Cutolo M, Tanaka Y. The role of nailfold videocapillaroscopy in patients with systemic sclerosis. Immunol Med. 2018;41:113-9.

39. Jinjuvadia R, Liangpunsakul S. Association between metabolic syndrome and its individual components with viral hepatitis B. Am J Med Sci. 2014;347:23-7.

40. Fatini C, Mannini L, Sticchi E, Rogai V, Guiducci S, Conforti ML, et al. Hemorheologic profile in systemic sclerosis: role of NOS3- 786T> $\mathrm{C}$ and $894 \mathrm{G}>\mathrm{T}$ polymorphisms in modulating both the hemorheologic parameters and the susceptibility to the disease. Arthritis Rheum. 2006;54:2263-70.

41. Soydinc S, Turkbeyler IH, Pehlivan Y, Soylu G, Goktepe MF, Bilici M, et al. Mean platelet volume seems to be a valuable marker in patients with systemic sclerosis. Inflammation. 2014:37:100-6.

42. Borbásné Farkas K, Szabo A, Lóránd V, Sarlos D, Minier T, Prohászka Z, et al. Clinical usefulness of measuring red blood cell distribution width in patients with systemic sclerosis. Rheumatology (Oxford). 2014;53:1439-45.

43. Shafer SL, Dexter F. Publication bias, retrospective bias, and reproducibility of significant results in observational studies. Anesth Analg. 2012;114:931-2. 